\title{
CRITICAL RADII FOR ALPHA-PARTICLE ELASTIC SCATTERING
}

\author{
M. LOZANO and G. MADURGA \\ Department of Atomic and Nuclear Physics, University of Seville, Spain \\ and \\ P.E. HODGSON \\ Nuclear Physics Laboratory, University of Oxford, UK
}

Received 28 December 1978

The critical radii obtained from analyses of the elastic scattering of alpha-particles by a range of nuclei are found to be closely connected with the nuclear matter distributions obtained by summing the squares of single-particle wavefunctions. This provides a method of calculating the details of the optical potentials from the structure of the target nucleus.

A recent analysis by Badawy et al. [1] of the elastic scattering of alpha-particles by several isotopic sequences at energies around the Coulomb barrier showed that the differential cross section essentially determines the radius $R$ at which the nucleon density is $2 \times 10^{-3}$ nucleons $\mathrm{fm}^{-3}$. These radii are approximately proportional to $A^{1 / 3}$, but there are small systematic differences due to the structure of the nuclei concerned. In particular, the quantity $R A^{-1 / 3}$ is found to increase slowly through some isotopic sequences and to decrease through others. This may be understood qualitatively in terms of the shell structure of the nuclei, since we expect the nuclear radius to increase more rapidly when a major shell is just beginning to be filled than when it is nearly full.

A quantitative study of this effect may be made by calculating the nuclear density distributions of the nuclei from a suitable model. It is necessary that the calculated distributions are particularly reliable in the surface region, and this is the case for those obtained by summing the squares of the appropriate singleparticle wavefunctions since for each state the potential is adjusted to fit the nucleon separation energy.

The nuclear density distributions $\rho(r)$ were calculated from the single-particle wavefunctions $\psi_{i}$ in a Saxon-Woods potential $V(r)$ using the expression $\rho(r)=\sum a_{i}\left|\psi_{i}\right|^{2}$

where $a_{i}$ is the occupation number of the state $i$ and the sum runs over all the occupied states.

The potential $V(r)$ has the usual form

$V(r)=V_{\mathrm{c}}(r)+U f_{1}(r)+\left(\frac{h}{m_{\pi} c}\right)^{2} U_{\mathrm{s}} \frac{1}{r} \frac{\mathrm{d} f_{2}}{\mathrm{~d} r} L \cdot \boldsymbol{\sigma}$,

where $V_{\mathrm{c}}(r)$ is the Coulomb electrostatic potential (for protons only), and the form factor $f_{i}(r)=[1+\exp$ $\left.\left\{\left(r-R_{i}\right) / a_{i}\right\}\right]$ with $R_{i}=r_{i} A^{1 / 3}$.

The expression (1) is corrected for the finite size of the nucleons by folding in their own density distributions, taking into account the Darwin-Foldy term and the centre-of-mass motion. The Perey non-locality correction factor was applied to the wavefunctions, and the final set was orthogonalised by the GramSchmidt procedure. A detailed account of the method of calculation is given in ref. [2].

Ideally, the occupation numbers $a_{i}$ are determined by analyses of nucleon transfer and knock-out reactions and the parameters of the potential are adjusted to optimise the fit to the model-independent charge distributions, which are often known to considerable accuracy and to the measured single-particle centroid energies. These potentials are then used to calculate the 
nuclear matter distribution. In practice some of these data are not available, so some of the information has to be estimated from general knowledge of nuclear systematics. The various ways of doing this are described below.

The density distribution obtained in this way is then fitted in the tail region by the expression

$$
\begin{aligned}
\rho(r) & =\rho_{0}\left[1+\exp \left\{\left(r-R_{\rho}\right) / a_{\rho}\right\}\right]^{-1} \\
& \approx \rho_{0} \exp \left\{\left(R_{\rho}-r\right) / a_{\rho}\right\}
\end{aligned}
$$

to give the best values of the surface diffuseness parameter $a_{\rho}$. The radius $R$ at which the nucleon density is 0.002 is thus given by $0.002=\rho_{0} \exp \left\{\left(R_{p}-R\right)\right\}$ $\left.a_{\rho}\right\}$. Setting $\rho_{0}=0.14$ gives

$R=R_{\rho}+4.25 a_{\rho}$.

Now Badawy et al. [1] have shown that the real part of the alpha-particle potential can be obtained from the nuclear density distribution using the relation

$R(0.2)=R_{\rho}+5.54 a_{\rho}+2.37$,

where $R(0.2)$ is the radius at which the real potential is $-0.2 \mathrm{MeV}$.

Thus from eqs. (4) and (5)

$$
R(0.2)=R+1.29 a_{\rho}+2.37 \text {. }
$$

This expression enables our density distributions to be compared directly with the values of $R(0.2)$ obtained by Badawy et al. [1].

A preliminary calculation was made using overall parameters, and subsequently the results were improved by including as much information as possible from the nuclei of interest.

The first calculation [3] used the integral occupation numbers of the simple shell model and obtained the potential $U$ from a non-local potential of depth $83 \mathrm{MeV}$ and non-locality parameter $\beta=0.87$. The form factor parameters $r_{i}=1.236 \mathrm{fm}, a_{i}=0.52 \mathrm{fm}$. To take account of the variation of potential through the isotopic sequences an asymmetry potential of $30 \mathrm{MeV}$ was added. The parameters of the spin--orbit potential were $7 \mathrm{MeV}$, with $r_{2}=1.1 \mathrm{fm}, a_{2}=0.65 \mathrm{fm}$. The results of this calculation in fig. 1 already show the same overall trends as the radii obtained from alphaparticle scattering.

The second calculation used the occupation numbers of Beiner and Lombard [4] and the potential

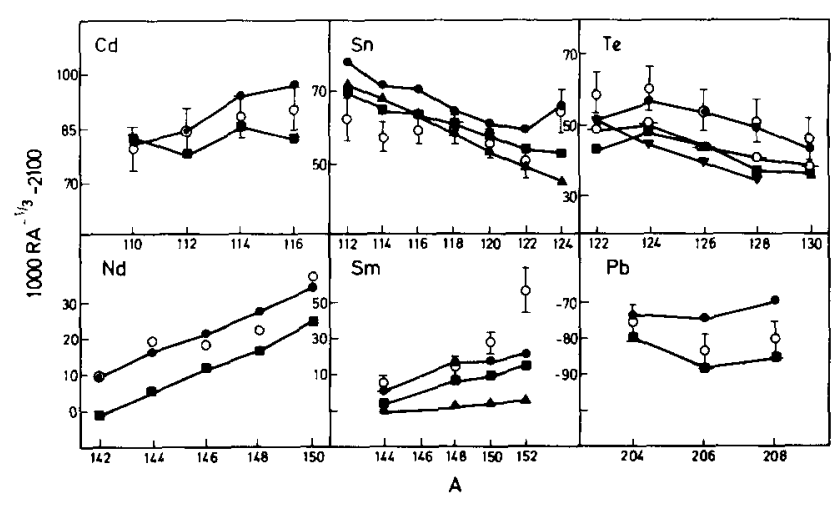

Fig. 1. Nuclear radius parameter as a function of $A$ for several isotopic sequences. 1 , experimental data of Badawy et al. [1] and of Tabor et al. [7] (for $\mathrm{Nd}$ ). Values calculated from nuclear density distributions $\bullet 1, \bullet 2, \vee 3, \circ 4, \bullet 5$.

depths were altered to fit his binding energies. The third calculation also used Lombard's results, but in addition used the experimental binding energies that are available for the isotopes $122,124,126,128 \mathrm{Te}$ [5]. The fourth calculation combined the integral occupation numbers and the experimental binding energies and the fifth used the potential of Bear and Hodgson [6]. In calculations 2, 3 and 4 the radius parameter $r_{1}=1.273 \mathrm{fm}$. These characteristics of the calculations are summarised in table 1 , and the corresponding results for the alpha-particle potential are compared with the values of Badawy et al. [1] and of Tabor et al. [7] (for Nd) in fig. 1 .

The comparison in fig. 1 between the experimental and calculated radii shows that the deviations from the overall $A^{1 / 3}$ variation are quantitatively reproduced. Use of the occupation numbers and potentials of Beiner and Lombard does not improve the fit. The best results are obtained with the potential of Bear and

Table 1

Nuclear density calculations

\begin{tabular}{llll} 
No. & $\begin{array}{l}\text { Occupation } \\
\text { numbers }\end{array}$ & $\begin{array}{l}\text { Binding } \\
\text { energies }\end{array}$ & Potential \\
\hline 1 & integral & - & non-local \\
2 & Beiner-Lombard & Beiner-Lombard & non-local \\
3 & Beiner-Lombard & (experimental) & non-local \\
4 & integral & (experimental) & non-local \\
5 & integral & - & Bear \\
\hline
\end{tabular}


Hodgson, which has been fitted to a wide range of experimental data.

This work establishes a direct connection between the nuclear matter distribution and the alpha-particle optical potential and provides a way of calculating the nuclear structure effects in the potential for energies around that of the Coulomb barrier.

The elastic scattering of particles by nuclei becomes increasingly sensitive to the nuclear surface as the mass of the particle increases. Thus nuclear structure effects of the type studied here are not found for nucleons, are appreciable for alpha-particles and are likely to be even more pronounced for heavy ions. Thus including information from the calculated nuclear densities should improve heavy ion optical potentials, and work along these lines is in progress.
Financial help from the British Council and from the Instituto de Estudios Nucleares, Madrid; is acknowledged.

\section{References}

[1] I. Badawy et al., Phys. Rev. C17 (1978) 978.

[2] F. Malaguti, E. Verondini, A. Uguzzoni and P.E. Hodgson, Nucl. Phys. A297 (1978) 287.

[3] M. Lozano, G. Madurga and P.E. Hodgson, Proc. Intern. Conf. on Nuclear interactions (Canberra, 1978).

[4] M. Beiner and R.J. Lombard, Ann. Phys. 86 (1974) 262.

[5] M. Conjeaud, S. Harar, M. Caballero and N. Cindro, Nucl. Phys. A215 (1973) 383;

R.L. Auble, J.B. Ball and C.B. Fulmer, Nucl. Phys. A116 (1968) 14

[6] K. Bear and P.E. Hodgson, J. Phys. G4 (1978) L287.

[7] S.L. Tabor, B.A. Watson and S.S. Hanna, Phys. Rev. C14 (1976) 514. 